\title{
Measurement of an Occupant's Lateral Motion by Using the Inertial Measurement Unit
}

\author{
Sunwoong Kim ${ }^{1 *}$, Yong-Man Kim¹, Yoon Hyuk Kim² and Won-Man Park ${ }^{2}$ \\ ${ }^{1}$ Hyundai Transys, Gyeonggi-Do, South Korea \\ ${ }^{2}$ Kyung Hee University, Gyeonggi-Do, South Korea \\ *Corresponding Author: Sunwoong Kim, Hyundai Transys, Gyeonggi-Do, South Korea.
}

Received: May 14, 2019; Published: June 12, 2019

DOI: $10.31080 /$ ASOR.2019.02.0063

\begin{abstract}
In recent years automotive seat comfort is one of a major factor both a researcher/engineer and a consumer. One of big issue is how to measure and analyze what consumers feel on a vehicle seats.

At first, it was required to measure how consumers' feel about what they feel while sitting on vehicle seats. Actually, there are some useful quantitative measurement methods for seating comfort SLD (Static Load Deflection), hardness profile, Vibration transmission, and impact damping test as well. However, all these evaluation methods focus on kinds of mechanical properties of vehicle seat itself. There were just few methods which are able to measure a relationship between vehicle seats and occupants like as BPD (Body Pressure Distribution). But these methods concentrated on the measuring the mechanical changes without occupant's contexts.

The needs of appropriate support while driving will be increased as improvement of technologies. The many drivers' want to feel their high vehicle performance and speed the better appropriate support will be provided by vehicle manufacturers. This is the second reason why we try to develop a new evaluation method for lateral support. It was required good evaluation method not only seat mechanical properties but also occupants motions while various driving situation.

There have been just few studies from the perspective of vehicle seat comfort, especially focus on drivers' behavioral aspects, even though the vehicles were made for convenience of human being.

These are the reasons that led us to start developing a new evaluation method from the perspective of occupants' behavioral study. The results of our study will be helpful to understand vehicle occupant context. And, it will be useful to not only develop a comfortable vehicle seat but also autonomous vehicle.
\end{abstract}

Keywords: Lateral Support; Vehicle Seat; Occupants Comfort; Driver Motion; Objective Measures for Comfort

\section{Introduction}

Seat lateral supports, which affect an occupant's comfort while driving, regarded as an importance matters that comfort consist of. Lateral supports are required under cornering and passing situation to restrict occupants' lateral movement for safe and comfortable driving. From these reasons, automotive seat system designers are to speak of their movement of better comfort. It is required to quantify the occupant side-way movements for better understanding of their behavior $[1,2]$.

With respect to lateral support, there were just a few relative researches. K. Noh conducted a study to the developing an ergonomic driver model based on human factors and its simulation environment from the perspective of driving task itself. K. Noh tried to figure out driver's physical recognition model for better understanding of decision make process [3]. Driver performance with limited-access highways characterized by directly quantifying the inputs to and outputs from the vehicle by J. Zhou. Various vehicle movement parameters were mainly considered as driver inputs and vehicle outputs from the perspective of vehicle operating - steering wheel angle, throttle position, brake reaction time, lateral position of the vehicle, and so on [4]. Almost all the further driver's behavioral studies focused on the driving tasks and vehicle response/movements without understanding the occupants' behavioral characteristics. 
Hardness profile, which measured a deflection through the overall range of both seat cushion and back with $50 \mathrm{~mm}$ cylinder under certain force, used to define and clarify his hardness distribution at insert area, uneven irritation feeling, and the hardness of the seat bolster as one of important factors that affect seat lateral support.

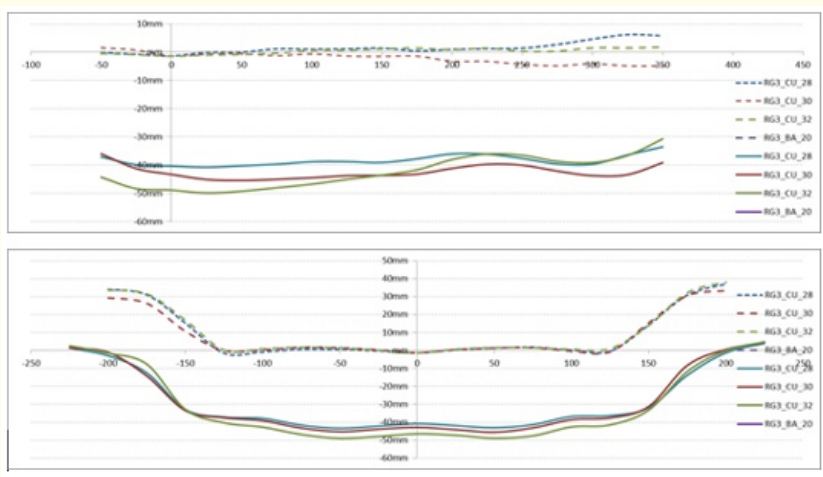

Figure 1: Examples of the measured hardness profiles with different seats.

BPD (Body pressure distribution), also, is one of famous methods to try to define and clarify how an automotive seat provide a lateral support under both static and dynamic driving situation. However, these methods have serious limitations to define a lateral support of the automotive seats. The former was more focused on a static situation, even thought, the most lateral support issues related with the dynamic situation. The later measured a reactional force on a vehicle seats against occupants from the vehicle seats. However, both automotive engineers and researchers are eager for understanding automotive occupants' behavior under cornering and passing.

Consequently, the appropriate measurement methods for understanding the automotive occupants' were required to both automotive engineers and researchers.

This paper will present a new method for observing occupants' motion by using the Inertial Measurement Unit (IMU) under actual driving. Newly proposed measurement method will used to develop a new comfort test method.

Tests methods

\section{Proving the IMU sensor's validity}

An optical motion analysis system was used to verify the IMU sensor's validity with simple laboratory tests.
As shown in Figure 2., installed eleven sensors are observing as follow;

- Head: Three optical sensors and one IMU sensor

- Upper Body: Three optical sensors and one IMU sensor

- Lower Body: Two optical sensors and one IMU sensor

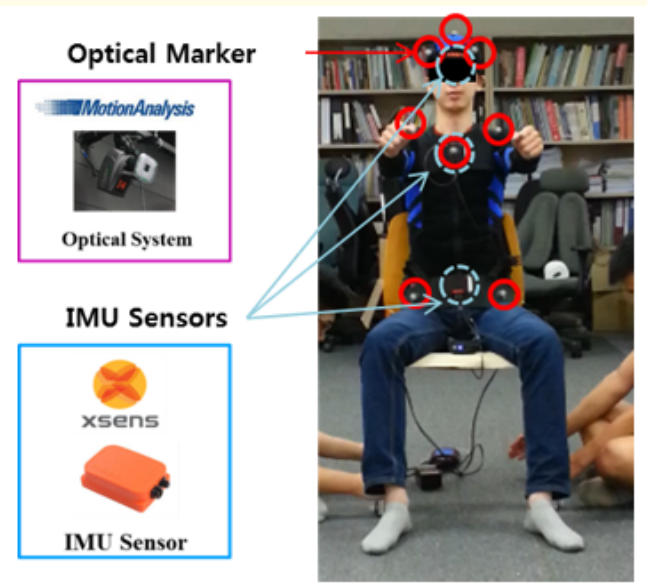

Figure 2: Eight Optical markers and three IMU sensors were installed to subject's body.

Left/right-side leaning motions were simulated by two facilitators. And, the motion was simultaneously recorded by each two types motion sensors to compare its validity.

\section{Measurement of a driver motion}

To develop measurement methods for a driver's upper body motion two driving sessions conducted. The first session, as a pilot study, focused on testing an appropriateness of using the IMU sensor system to measure driver motion and clarifying a sensor installation on both driver and vehicle (seats). The last session conduced to figure out a driver's motion while actual driving.

\section{A pilot test}

The IMU sensors installed on a driver's body (torso and pelvic) and vehicle seats (cushion and back) to observe his motion while tests driving. The sensors on the seats were used to reference for calculating the relative angles of each pelvic and torso movements. Two channels of body Pressure were measured at the same time to analyze driver's body movements.

Tests driving course designed as simple to figure out a validation of the IMU sensor (Figure 3). The course consists of 1) above $70 \mathrm{~m}$ straight way to reach $30 \mathrm{~km} / \mathrm{h}, 2$ ) radius $12.5 \mathrm{~m}$ of circled way to evaluate cornering motion of a driver with maintaining the $30 \mathrm{~km} / \mathrm{h} .3$ ) and, return way to start point. 

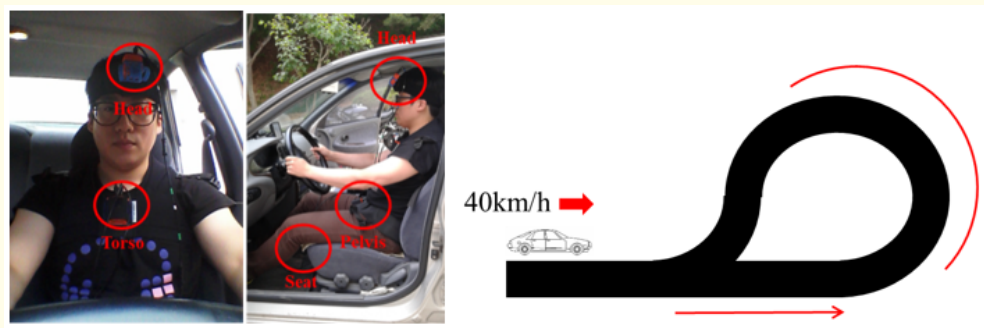

a. sensor installation $b$. tests driving course

Figure 3: Three IMU sensors installed on driver's head, sternum and center of ASIS (Anterior superior iliac spine) and two of vehicle seats - front area of seat cushion as a reference of vehicle movement and backboard of seat back.

Driving test on the road

The selected tests roads included various cornering situation small/large single cornering and multi-left and right turns. It took less than one minute with around $50 \mathrm{kph}$. Sensors were set the same location but head. All sensors initialized before start driving to prevent any sensor drift. The movement of driver was calculated by gyro-sensors. A numerical calculation, as linear calibration, were implemented to compensate the sensor drift effect through measure the initial and the last terrestrial magnetism sensor value. It was selected each one vehicle from BIC (Best in Class) and WIC (Worst in Class) as tests vehicles with three volunteered subjects. The vehicle, as BIC and WIC, selected by APEAL (Automotive Performance, Execution, and Layout) study, which conducted by JDPA (JD Power and Associates) [5]. The volunteered subjects were consisted with various age bands (20s to $40 \mathrm{~s})$, body sizes $(167 \mathrm{~cm}$ to $180 \mathrm{~cm} ; 65 \mathrm{~kg}$ to $85 \mathrm{~kg}$ ), and driving careers ( 5 years to more than 20 years).

\section{Results of the Studies}

IMU sensor's validity tests results

Relative subject motion to chair was calculated to compare measured movement both IMU sensor and optical motion analysis system. As results of comparison, there were no big differences between the two measures - pelvic motion $<1.5$ degrees, torso motion $<2.0$ degrees. These observed differences assumed that caused from measurements - installing each sensor, calculating the data, and so on- and own error rates of each equipment. However, it is enough to consider IMU sensor as an appropriate measures for observing vehicle occupants motion.
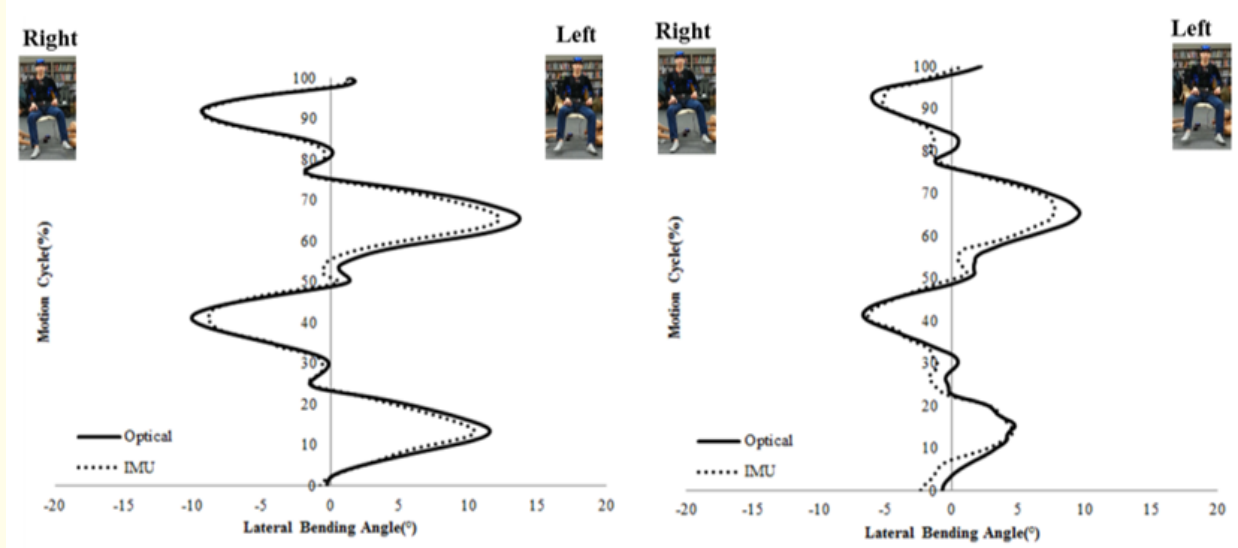

a. Relative lateral bending - Pelvic b. Relative lateral bending - Torso

Figure 4. Relative lateral bending angle of pelvic was shown at Figure 4-a. There were maximum 1.5 degree of angle differences between the two observations. Relative lateral bending angle of torso was shown at Figure 4-b.

There were maximum 2 degree of angle differences between the two observations. 


\section{Results from measurement of driver's motion}

\section{A Pilot tests}

It was observed that a slight flexion motion alongside vehicle start (Figure 4-b). The movement of driver's body was starting to set with making 'J' turns up to 17.6 degrees. The opposite side movements was occurred by turning into the straight way.
According to the pilot study, a driver's head movements had the largest reactions against the movements of a vehicle. However, it obviated the need for considering as independent variables due to unspecific head movements that have less related with test drive.
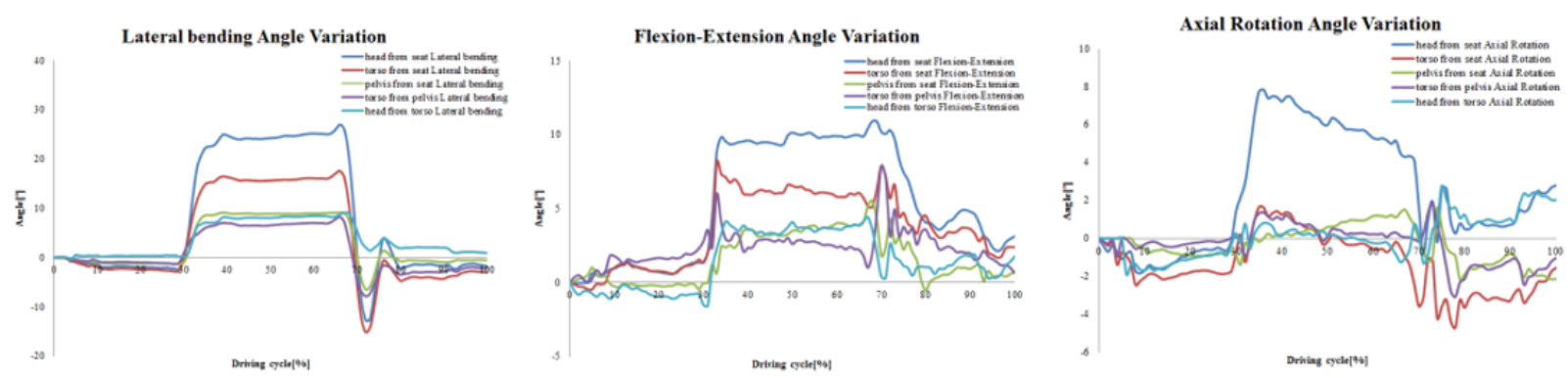

a. Lateral bending angle b. Flexion-extension angle c. Axial rotation angle

Figure 5: Measured data translated to driver's motion - a. lateral bending, b. flexion-extension and c. axial rotation.

The measured pressure data set were divided into two regions - left and right side - for each mat on seat cushion and back. It seemed that the possibilities to measure and analyze driver's lateral movements under various driving situation was quite high as enough to use an appropriate measures. However, there founded some problems. Major problem was late response rate. The IMU sensors followed by both observed mean and peak pressure changes. Another problem was low level of pressure range. Most of appropriate pressure measurement system in auto industries have less than $34 \mathrm{kPa}$. However, in many cases, it will be required more higher level of pressure ranges in various driving environments and situations like as large body sized occupants, wallet in pocket, and heavy driving situation as well.
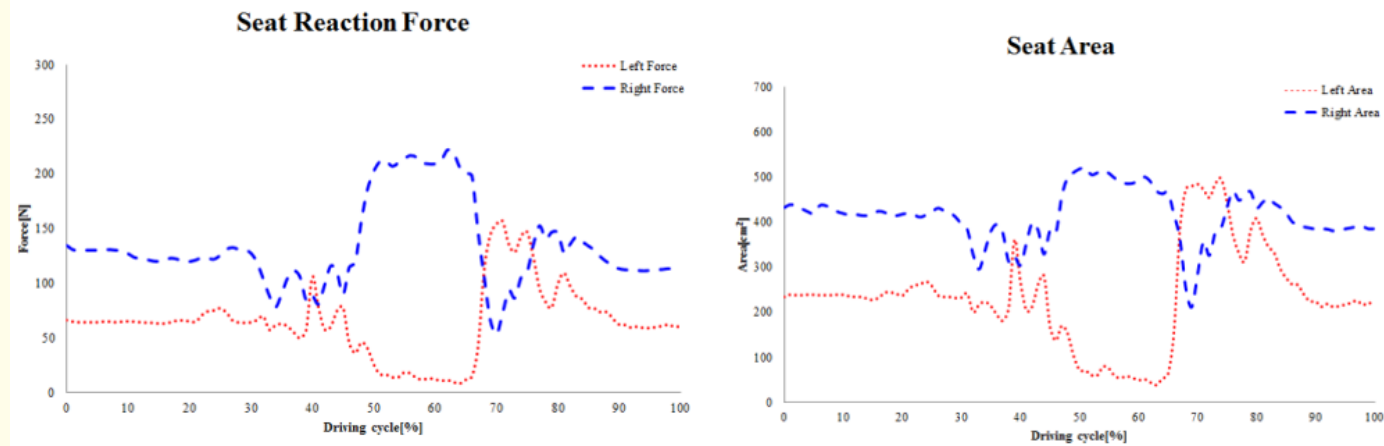

a. Seat mean pressure b. Seat peak pressure

Figure 6: Mean and peak pressure was calculated from measured body pressure. 
Driving tests on the roads

The roads tests conducted with back and forth from start point to the end. Each odd/even numbers of trials showed the same way of tests drive mode. For example, each try \#1, 3, 5, 7, and 9 means tests drive mode from start point to the end. But the even numbers mean tests drive mode which going back to start point.

\begin{tabular}{|c|c|c|c|c|c|c|}
\hline \multirow[b]{2}{*}{ Class } & \multicolumn{3}{|c|}{ Vehicle A (BIC) } & \multicolumn{3}{|c|}{ Vehicle B (WIC) } \\
\hline & $\begin{array}{c}\text { Left lateral bending } \\
\text { angles (1) }\end{array}$ & $\begin{array}{c}\text { Right lateral } \\
\text { bending angles } \\
\text { (2) }\end{array}$ & $\begin{array}{c}\text { Bending Angle } \\
\text { (1) + (2) }\end{array}$ & $\begin{array}{c}\text { Left lateral } \\
\text { bending angles } \\
\text { (1) }\end{array}$ & $\begin{array}{c}\text { Right lateral } \\
\text { bending angles } \\
\text { (2) }\end{array}$ & $\begin{array}{c}\text { Bending Angle } \\
\text { (1) + (2) }\end{array}$ \\
\hline Try \#1 & 2.5 & 10.8 & 13.3 & 16.9 & 11.8 & 28.7 \\
\hline Try \#2 & 11 & 8.5 & 19.5 & 15.7 & 14.4 & 30.1 \\
\hline Try \#3 & 1.9 & 8.8 & 10.7 & 13.4 & 11.2 & 24.6 \\
\hline Try \#4 & 8.7 & 2.1 & 10.8 & 21.2 & 7.4 & 28.6 \\
\hline Try \#5 & 8.5 & 8.4 & 16.9 & 5.1 & 12.3 & 17.4 \\
\hline Try \#6 & 10.6 & 4.5 & 15.1 & 12.4 & 9.2 & 21.6 \\
\hline Try \#7 & 4.9 & 11.4 & 16.3 & 10.9 & 13.2 & 24.1 \\
\hline Try \#8 & 12.9 & 4.4 & 17.3 & 13.7 & 8.3 & 22 \\
\hline Average & \multicolumn{3}{|c|}{15.0} & \multicolumn{3}{|c|}{24.6} \\
\hline Stdev. & \multicolumn{3}{|c|}{3.16} & \multicolumn{3}{|c|}{4.32} \\
\hline
\end{tabular}

Table 1: Lateral bending angles of upper body.

In case of vehicle $B$, it showed that the large amount of lateral bending of upper body as expected. The averaged lateral bending angles were up to $25.6 \pm 3$.8 degrees for vehicle $B$, whereas vehicle A were less than $15.7 \pm 3.2$ degrees. The range of bending angles for vehicle A (8.8 degrees) was showed 31\% less than vehicle B's (12.7 degrees).

\section{Discussions and Conclusions}

Throughout this study, two types of driver's motions observed during a cornering - which showed counter balance or not. These behavioral activities were depends on their driving tendency.
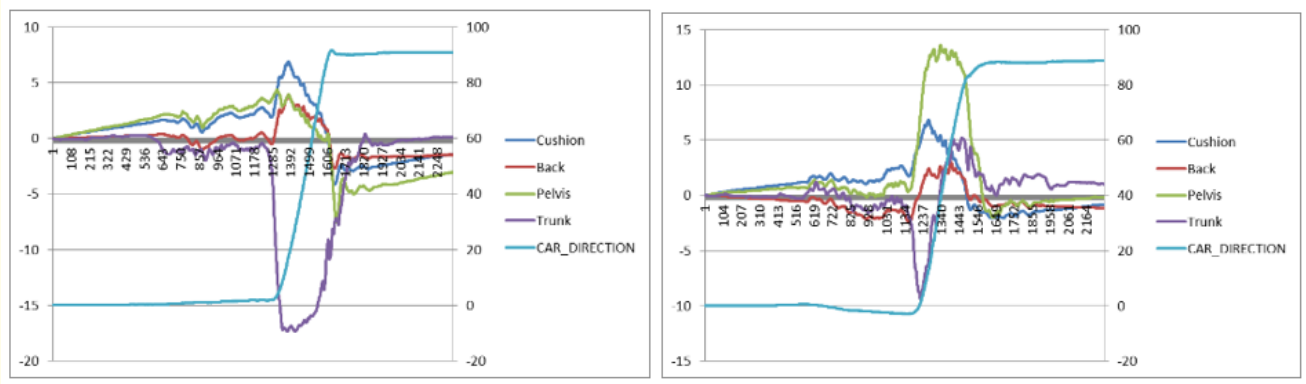

a. None counter balance motion b. Counter balance motion

Figure 7: It showed a different upper body movements according to whether do counter balance motion or not.

In general, human body tends to keep the projected COM (Center of Mass) on the ground into their base of support (BOS) to make a stable position. On the same principles, driver wanted to locate their projected COM into their BOS. 


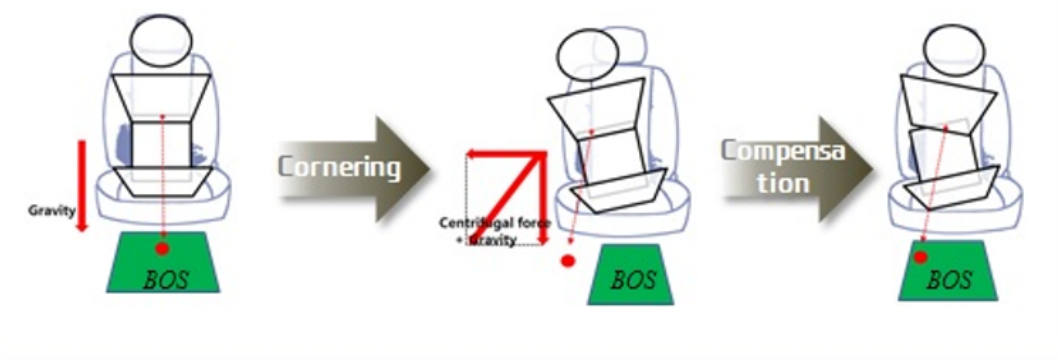

Figure 8: It was observed that driver's upper body movements to opposite side against lower body to maintain stable posture while cornering or changing the lane.

A big difference of these two cases is the movements of their upper body and pelvic. A driver who do not have counter balance motion showed more large pelvic movement when starts cornering. In this case, the putative reason is that the driver do not have appropriate physical preparation on the cornering even though he already have their psychological readiness for making turn. It leads to large amount of upper body bending motions compare to his pelvic movements against a driver who having a counter-balancing driver (Figure 7 and Figure 8).

It was founded that asymmetrical motion of occupant while cornering. It caused from behavioral differences between left and right lower leg. It, also, occurred from different shape and mechanical properties of the vehicle seats and asymmetrical vehicle interior as well.

This study suggested that a new measurement methods for driver's motion during driving or testing and verified the suggested methods. One of limits of this study was small sample size.
Bibliography

1. DA Coelhoa and S Dahlmanb. "A pilot evaluation of car seat side support: Leading to a redfinition of the problem". International Journal of Industrial Ergonomics 24.2 (1999): 201-210.

2. F Colomobet., et al. "Motion Cueing: What is the impact on the driver's behavior?" DSC 2008 Europe (2008): 171-182.

3. K Noh., et al. Development of Ergonomic Driver Model Considering Human Factors, SAE World Congress, paper no. 2007-01-3584 (2007).

4. J Zhou., et al. "Characterization of the Lateral Control Performance by Human Drivers on Highways, SAE World Congress, paper no. 2008-01-0561 (2008).

5. JD Power. The J.D. Power U.S. Automotive Performance, Execution and Layout Study (2015).

\section{Volume 2 Issue 7 July 2019}

(C) All rights are reserved by Sunwoong Kim., et al. 